\title{
Related Factors of the Influence on Mental Symptoms of the Recruits in the Basic Military Training*
}

\author{
Li Hong-zheng, Lei Mei-ying, Zhao Dong-hai, Zhao Li-qiong, \\ Liu Geng, Zhou Hong-kui, Qin Mei, Li Jie-feng, Wen Jian, \\ Huang Pin-de, Li Yi, Wang Chuang, Wang Zhou-ran \\ The 303rd Hospital of PLA, Nanning, China
}

\begin{abstract}
The objective of the study is to explore the psychosocial characteristics of recruits for mental health education during the basic military training. A total of 1,366 male recruits were assessed during the basic military training. The psychosocial characteristics, such as effects of LE (life events), mental symptoms, personality trait coping style and social support of the recruits were assessed with LEI-CSLA (LE Inventory for Compulsory Servicemen of Land Army), SCL-90-R, EPQ (Eysenck Personality Questionnaire), SCSQ (Simple Coping Style Questionnaire) and social support scale respectively. Statistical analyses, such as multiple stepwise analyses, interaction analyses and path analysis, were used. Neuroticism trait, negative coping style, objective social support and LE were significant factors in determining the score of mental symptoms, accounted for $64 \%$ of the total variance, the absolute value of $\beta$ of each variable was 0.09-0.48. LE and neuroticism had direct effect on SCL-90 symptom index. LE, mental symptoms, personality trait, coping style as well as social support should be taken into consideration when psychological intervention and mental health education are needed in service for recruits in the basic military training.
\end{abstract}

Keywords: mental health, health education, recruits, basic military training

\section{Introduction}

Basic military training consists of highly regimented training in the context of fairly extreme

\footnotetext{
*Acknowledgments: This study was supported by the Project of Mental Health Research of PLA (Grant No.12XLZ204), the Project of Science and Technology Research in Medicine of PLA (Grant No.CWS11J278), the Natural Science Foundation of Guangxi (Grant No. 0991289) and the Guangxi Health Department Project (Grant No. Z2009404).

Li Hong-zheng, professor, postdoctor, Mental Health Center, The 303rd Hospital of PLA.

Lei Mei-ying, associate professor, Mental Health Center, The 303rd Hospital of PLA.

Zhao Dong-hai, professor, The 303rd Hospital of PLA.

Zhao Li-qiong, associate professor, Mental Health Center, The 303rd Hospital of PLA.

Liu Geng, Ph.D., Mental Health Center, The 303rd Hospital of PLA.

Zhou Hong-kui, Ph.D., Mental Health Center, The 303rd Hospital of PLA.

Qin Mei, Mental Health Center, The 303rd Hospital of PLA.

Li Jie-feng, Ph.D., Mental Health Center, The 303rd Hospital of PLA.

Wen Jian, Ph.D., Mental Health Center, The 303rd Hospital of PLA.

Huang Pin-de, Ph.D., Mental Health Center, The 303rd Hospital of PLA.

Li Yi, Ph.D., Mental Health Center, The 303rd Hospital of PLA.

Wang Chuang, Mental Health Center, The 303rd Hospital of PLA.

Wang Zhou-ran, Mental Health Center, The 303rd Hospital of PLA.
} 
psychosocial stressors and recruits suffered from rigorous disturbance in mind. In our research, the identification of the related factors may help us allow for the future development of brief primary prevention interventions with which the related factors of vulnerable individuals can be modified, thus preventing the development of clinical symptoms.

Military stressors of recruits abroad included sleep deprivation, anticipatory stress, congestion of inhabitancy and constraint of life habit. It was suggested by general people that LE (life events), social support and neurotic trait were the main factors affecting mental status, but it was not confirmed that the conclusions were applicable for recruits.

In this report, we will examine the effects of LE on mental symptoms in a model including some other factors, for example, social support, neurotic trait and coping style. The purpose of this study is to examine further the association between retrospective self-reports of LE and mental symptoms of recruits, the direct and indirect effects of LE on mental status and the mediate factors through which the effects of LE were made on mental status.

\section{Methods}

\section{Participants}

A total of 1,366 male recruits in Land Army participated in our studies after permission was granted. Their mean age was 18.81 years $(S D=1.08)$. Education level of most of the participants was 8-13 years. No recruits reported that they had been currently married. The sample was predominantly Han nationality (92.4\%), and the remainder indicated they were from other ethnic groups.

\section{Measures}

Effects of LE. It was assessed with LEI-CSLA (LE Inventory for Compulsory Servicemen of Land Army). The 93-item LEI-CSLA uses a 5-point response format to measure the effects of LE on the mental status of recruits and has higher internal consistency and test-retest reliability, it likewise has a sound record for validity in terms of factors analyses and testing in concurrent validity.

Mental symptoms. General psychiatric symptoms were assessed using the SCL-90-R (revised 90-item symptom checklist). The SCL-90-R is a self-report measure for assessing 90 symptoms experienced during the past seven days, rating on a 5-point scale from 0 (“Not at all”) to 4 (“Extremely”). Symptom index, also the total score dividing 90, was viewed as the level of mental symptoms.

Personality trait. Participants had completed the EPQ (Eysenck Personality Questionnaire) for adults revised for Chinese. The EPQ has 88 items assessing four dimensions of personality trait (neuroticism, extrovision or introvision, psychoticism and lie) and each item was scored in a dichotomous manner: $1=$ "Endorsement or yes"; 0 = "Non-endorsement or no".

Coping style. The SCSQ (Simple Coping Style Questionnaire), which includes 20 items attributing to two 
dimensions (the positive coping style and the negative one), had been distributed to 1,091 recruits to assess their habitual coping skill.

Social support. Social support was assessed by SSS (social support scale) that contains 12 items compiled for general people, few items were modified in some sort for the recruits in our study. The 12 modified items ascribe to three dimensions (the subjective social support, the objective one and the support utility).

\section{Procedure}

One thousand three hundred and sixty six participants sampled randomly from a recruits unit in Land army who completed self-report questionnaires at the end of the basic military training. All data were collected in groups and each group consisted of 20-30 recruits within three days when we get the study permission of the military administration department.

\section{Date Analysis and Statistic}

Dates were expressed as $\bar{X} \pm s$. Multiple stepwise analysis, interaction analysis and path analysis were used in our studies. All the statistics were completed by computer and SPSS 10.0 (statistical package for social science) were used in statistics.

\section{Analyses and Results}

\section{Multiple Stepwise Analyses}

Multiple stepwise analyses of data from the 1,366 recruits were carried out with symptom index of SCL-90 taken as dependent variables, and the following factors, including four dimensions of EPQ, two dimensions of coping style and three dimensions of social support and LE, were taken as independent variables. The results showed that the overwhelming influence on the score of mental symptoms was the neuroticism trait (the score of $N$ dimension) of recruits, although NCS (negative coping style), objective social support and LE were also significant factors in determining the score of mental symptoms. All the four significant variables entering the regression equation accounted for $64 \%$ of the total variance, the absolute value of $\beta$ of each variable was $0.09-0.48$. In order of the size effect, the significant $\beta$ coefficients were 0.43 for $\mathrm{N}$ (neuroticism), 0.37 for LE, 0.16 for NCS, and -0.09 for objective social support.

\section{Interaction Analyses}

New interaction variables, including interaction 1, interaction 2 and interaction 3, were developed with the score of LE multiplied by that of neuroticism, NCS and objective social support in sequence. Linear regression by enter methods was carried out with symptom index of SCL-90 taken as dependent variables, the four significant variables in stepwise analysis as independent variables in layer 1 , and the three interaction variables as those in layer 2. The results showed that only interaction 1 between LE and neuroticism had direct effect on SCL-90 symptom index, although this is not the case for the other two 
dummy variables of interaction 1 and interaction 2. All the five variables (the four significant variables in stepwise analysis and interaction 1) accounted for $66.4 \%$ of the total variance, and the $R^{2}$ change was also significant at $p<0.01$.

\section{Path Analysis}

For the five significant variables (the four significant variables in the stepwise analysis above and the interaction 1), we assumed that LE could affect mental symptoms directly and (or) indirectly, the mediate factors included personality, social support, coping style, or (and) interactions. Based on the hypothesis mentioned above, a path diagram was developed for further path analysis.

Path analysis is concerned with erecting a casual structure compatible with the observed date, the path diagrams are the qualitative representations of a set of structural equations relating the variables under study and the structural equations implied by a path diagram are all linear equations. In order to get the pass coefficients of each independent variable to dependent one, we used linear regression by enter methods to carry out analysis with anyone of the five significant independent variables taken as dependent variable $\left(Y_{j}\right)$ and the four left as independent variables $\left(X_{i}\right)$.

According to the results of the path analysis carried out above, linear regression with enter methods were carried out again with anyone of the six variables (including the symptom index of SCL-90) taken as dependent variable $\left(Y_{j}\right)$ and the variables, only with significant path coefficients, as independent variables $\left(X_{i}\right)$. Based on the data got from linear regression by enter methods for six dependent variables, a path diagram of the relationship between LE and the other factors was erected.

\section{Discussion}

There are many studies on the relationship between LE and diseases, but still now we cannot ascertain the causality of them. Some investigations have shown that the correlation level of the LE and diseases was lower (about 0.12 ), so some scholars suggested that we should strengthen the research on the vulnerability factors and amelioration ones related to disease. In this aspect, many factors (for example, personality, social support, coping style and LE) were concerned.

Our results show broadly similar findings to previous studies that have been noted by Thoits (1983) and Goyne (1991). Regression analyses for mental symptoms of recruits in our studies showed that four variables (EPQ-N, LE, NCS and objective social support) entered the regression equation, it indicated that the four factors had direct effects on the mental symptoms of recruits, the absolute value of $\beta$ coefficients ascertained that the overwhelming influence on the mental symptoms score was N, although LE, NCS, and objective social support in sequence are also significant factors in determining mental symptoms. At the same time, according to the quality of $\beta$ (positive or negative), we could draw a conclusion that three factors including $\mathrm{N}$, LE, and NCS may be the vulnerable factors for mental symptoms, while objective social support is the amelioration 
one.

There was also general acceptance that neuroticism represents a genetically influenced trait underlying the vulnerability to develop mental symptoms (Duggan et al., 1995). This has been largely based on the results of twin and family studies, which indicated that neuroticism was heritable and that the score was stable over time (Santor et al., 1997). Therefore, in the viewpoint of mental intervention for recruits, three variables (including LE, NCS and objective social support) might be taken as factors that can be intervened, although neuroticism was not the case even, if it had overwhelming effects on mental symptoms.

The relationship between excess adverse events and depression could be mediated by aspects of personality, such as neuroticism and extraversion. For example, neurotic traits could lead to an individual being excessively concerned about the occurrence of an adverse event (i.e., threat perceiving).

In our studies, the interaction analysis indicated that interaction of LE and neuroticism had direct effect on SCL-90 symptom index, that is to say, neurotic trait could regulate the severity of mental symptom in different levels of LE, not only the LE and the neuroticism themselves, but also their interaction could affect the mental symptoms of recruits. It suggested that when psychosocial intervention is needed for recruits' mental symptoms, we should pay attention to the two factors at the same time.

A path analysis enables us to evaluate the direct effect of one cause on an effect and its indirect effect via other causes. Our studies showed that LE affected mental symptoms not only in direct ways, but also in indirect ways, neuroticism and NCS, but not objective social support, acted as intermediate variables to affect mental symptoms. It also suggested that high neuroticism and NCS might exert some negative effects on mental symptoms, whereas objective social support exerted protective effects from the symptoms.

The identification of the related factors may help us allow for the future development of brief primary prevention interventions with which the related factors of vulnerable individuals can be modified, thus preventing the development of clinical symptoms.

Of course, the findings of this study may be limited in terms of sample and the instruments applied. First, further improvement of the LEI-CSLA is warranted in the future, although it has been proved to be rather reasonable in psychometrics. Second, only one path diagram was erected based on the authors' comprehension of the relationships among those factors in our studies, there may be many path diagrams and further comparison is also required. Third, few intermediate factors were taken into account in the analysis of their effects on mental symptoms, further studies for more variables await.

\section{Conclusions}

It is commonly perceived that basic military training is both physically stressful to the new recruits and mentally stressful due to the need to adapt to a new environment. Rigorous military training is also thought to maybe lead to mental disorders and early separation of the recruits from military. In our study, symptoms of the 
recruits can be affected directly by neuroticism, LE, negative coping style, objective social support and the interaction between neuroticism and LE. LE can affect mental symptoms indirectly through mediate factors including neuroticism, objective social support, negative coping style, as well as the interaction between neuroticism and LE. Our research suggested that: (1) Early detection of the relationship of the psychosocial factors of recruits could be benefited to perform mental health education; (2) Psychological interference may be necessary for new recruits; and (3) The factors above should be considered for the development of the psychosocial screening instrument for recruits.

\section{References}

Avison, W. R., \& Gotlib, I. H. (1994). Stress and mental health contemporary issues and prospects for the future. Plenum Press.

Duggan, C., Sham, P., Lee, A., et al. (1995). Nurotisim: A vulnerability marker for depression, evidence from a family study. Journal of Affective Disorder, 35, 139-143.

Farmer, E., Redman, K., Harris, T., et al. (2002). Neuroticism, extraversion, life events, and depression. British Journal of Psychiatry, 181, 118.

Gold, M. A., \& Friedman, S. B. (2000). Cadet basic training: An ethnographic study of stress and coping. Military Medicine, 165, 147-152.

Goyne, J. C., \& Downey, G. (1991). Stress, social support and the coping process. Ann Rev Psychology, 42, 401-426.

Huang, Z. N. (1995). Medical multianalysis. China: Hunan Science and Technology Press.

Li, X., Gail, H., \& Qian, M. Y. (2001). Steps of the use of structural equation model and its applications in clinical psychology. Chinese Journal of Clinical Psychology, 9, 149

Li H. Z., Li, X. R., Lei, M. Y., et al. (2004). Development and psychometric properties of life events inventory for compulsory servicemen of land army. Chinese Journal of Clinical Psychology, 12, 234-236.

Park, B. G., \& Jones, P. B. (2001). Neurotics, life events and mental health: Evidence for person environment correlation. British Journal of Psychiatry, 178, 72-77.

Santor, D. A., Bagby, R. M., \& Joffe, R. T. (1997). Evaluating stability and change in personality and depression. Journal of Personality and Social Psychology, 73, 1354-1362.

Thoits, P. A. (1983). Dimensions of life events that influence psychological distress: An evaluation and synthesis of the literature. In Psychological stress (1st ed., p. 33). New York: Academic Press.

Wang, X. D., Wang, X. L., Ma, H., et al. (1999). Rating scales for mental health. China: Chinese Mental Health Journal Press.

Wu, M. L. (2000). Statistic practice of SPSS. China: Chinese Railway Press.

Xu, C. T. (1997). Role of the relationship among social support, personality, life events and mental health. Chinese Journal of Clinical Psychology, 5, 91-94. 